\section{Initiation of Warfarin in Hospitals}

Editor - Warfarin is a potential life saving agent but the risks of warfarin therapy are nevertheless substantial so appropriate dosage titration is mandatory. The individualization of warfarin therapy has traditionally been empiric but because significant numbers of patients are either under-treated or over-treated, 1,2 methods have been devised to predict optimal warfarin doses. This is because it has been noted that the eventual dose required varies widely between apparently similar people, and the consequences of either underanticoagulation or over-anticoagulation are clinically important. ${ }^{3}$ Also, the time taken to initiate treatment and decide the correct maintenance dose by the traditional empirical method often takes several weeks.

Many models for the initiation and control of warfarin therapy have been proposed and I read with interest the dose prediction method of Caldwell et $\mathrm{al}^{4}$ published in the last issue of Clinical Medicine \& Research which explained 56\% of the variation in the maintenance dose. The dosing algorithm made use of genetic testing that is complex, only feasible in the United States, and not currently available to clinical practice. ${ }^{4}$ This reminded me of the pharmacodynamic model $^{5,6} \mathrm{I}$ had proposed in 1994 and later modified in 2001 with results that have been validated in two different cohorts 6 and translated into a simple nomogram for clinical use (figure 1). This method had then explained $73 \%$ of the variation in the maintenance dose and required no special test, and was very easy to implement and use. The fact that Caldwell et al were contemplating such a difficult system, given the availability of simpler yet similarly predictive systems, prompted me to write about this method.

To use this dose prediction method, an international normalization ratio (INR) is done at the time of admission on day 1 and then at 8 AM daily for days 2 to 4 and then less frequently thereafter. It should be pointed out that there exists the routine variability normally seen in clinical practice for both the administration of warfarin and sampling of blood for INRs, so these timings are not exact. It was aimed to administer warfarin $10 \mathrm{mg}$ at 1600 hours daily according to the nomogram on days 0 and 1 , and the INR is measured on days 0 to 2 at 0800 hours. The age of the patient and the INR at 0800 hours on day 2 are used to read off the patients' maintenance dose on the nomogram. If an INR is $>2.5$, this maintenance dose is held until the INR is down to 2.5. All patients are simultaneously commenced on intravenous unfractionated heparin according to the hospital protocol. Heparin is discontinued when the INR

Keywords: Warfarin, Anticoagulation, Nomogram, Internationa

normalization ratio (INR)

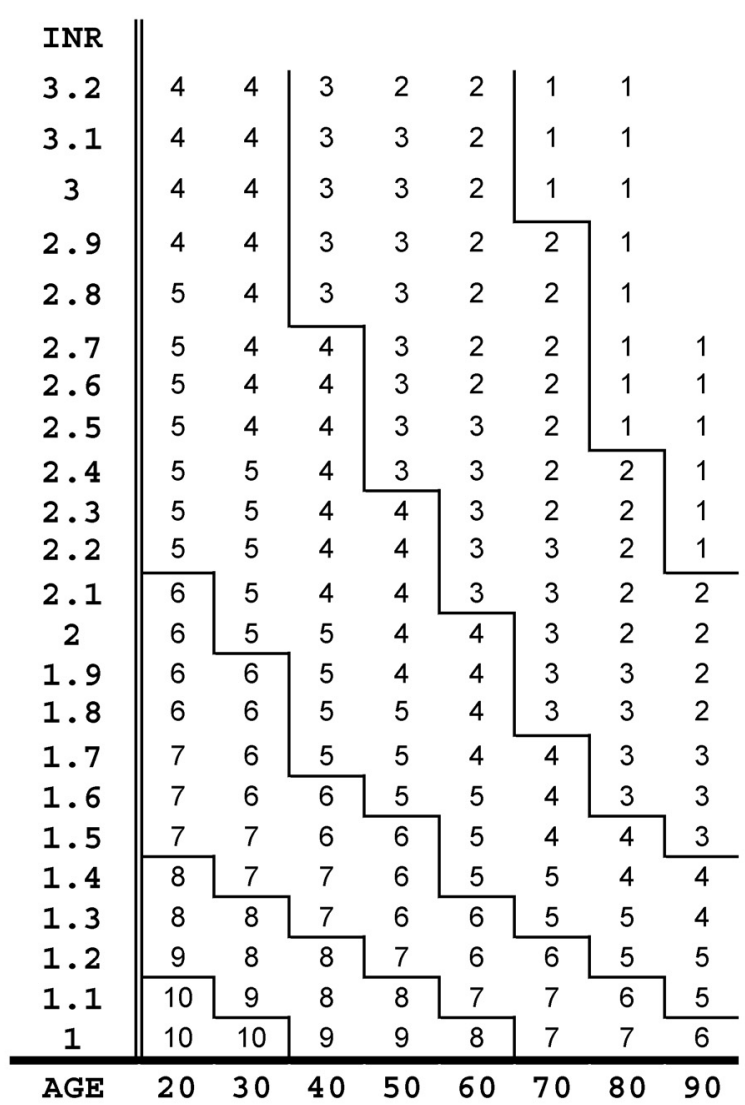

Figure 1. Nomogram for warfarin initiation.

is $\geq 2$. Follow-up is usually in the hospital's anticoagulation clinic run by the hematology service or medical outpatients. My experience with the use of this model for dose prediction in Kuwait has been excellent, and indeed in a random selection of 13 patients this model explained $97 \%$ of the interindividual variation in warfarin dose requirements.

With a simple predictive model such as this that explains $73 \%$ or more of the interindividual variability in warfarin maintenance dose requirements, I think it would be safe to conclude that the use of this model in the form of the included nomogram (figure 1) should make it less appealing to come up with more complex methods, such as that suggested by Caldwell et $\mathrm{al}^{4}$ of decreasing both the occurrence of either under-anticoagulation or over-anticoagulation, as well as the time taken to initiate treatment and decide the correct maintenance dose during the initiation of oral anticoagulation with warfarin in hospitals. It is also clinically feasible and very simple to implement. I would, therefore, like to bring this
Received: June 25, 2007

Revised: October 18, 2007

Accepted: October 25, 2007 
to the attention of physicians involved with anticoagulation who are contemplating other methods for the initiation of warfarin in hospitals.

\section{References}

1. Duxbury B. Therapeutic control of anticoagulant treatment. Br Med J (Clin Res Ed) 1982;284:702-704.

2. van den Besselaar AM, van der Meer FJ, Gerrits-Drabbe CW. Therapeutic control of oral anticoagulant treatment in the Netherlands. Am J Clin Pathol 1988;90:685-690.

3. Holford NH. Clinical pharmacokinetics and pharmacodynamics of warfarin. Understanding the dose-effect relationship. Clin Pharmacokinet 1986;11:483-504.

4. Caldwell MD, Berg RL, Zhang KQ, Glurich I, Schmelzer JR, Yale SH, Vidaillet HJ, Burmester JK. Evaluation of genetic factors for warfarin dose prediction. Clin Med Res 2007;5:8-16.

5. Doi SA. Pharmacodynamic optimization of warfarin therapy. Clin Pharmacol Ther 1994;55:597-601.

6. Doi SAR Pharmacodynamic optimization of warfarin therapy II. Am J Ther 2001;8:41-47.

Suhail A.R. Doi, PhD, FRCP

Department of Medicine (Endocrinology) Mubarak Al-Kabeer Teaching Hospital and Kuwait University, Kuwait sardoi@gmx.net 